\title{
Los Criterios de Evaluación del Aprendizaje en la Educación Superior
}

\author{
The Evaluation Criteria of Learning in Higher Education
}

\author{
Abel Federico Pérez Hernández* \\ Cristian Janet Méndez Sánchez ${ }^{* *}$ \\ Pedro Pérez Arellano*** \\ Héctor Manuel Yris Whizar****
}

\author{
*Universidad Juárez Autónoma de Tabasco. División Académica \\ Multidisciplinaria de Jalpa de Méndez. \\ Playas de Catazajá. Manzana 10, lote 8-B. Fracc. Playa Azul. \\ Cunduacán, Tabasco. México. C.P. 86690. \\ Tel. Cel. 9931936273 \\ abel.perez@ujat.mx \\ ${ }^{* *}$ Universidad Popular de la Chontalpa. \\ Playas de Catazajá. Manzana 10, lote 8-B. Fracc. Playa Azul. \\ Cunduacán, Tabasco. México. C.P. 86690. \\ Tel. Cel. 9933016254 \\ cristianjanetmendez@gmail.com
}

***Universidad Juárez Autónoma de Tabasco. División Académica de Informática y Sistemas.

Circuito Laguna la Mocha Lote 21, Manzana 4E. Fracc. Lagunas. Villahermosa, Tabasco. México. C.P. 86019.

Tel. Cel. 9932247563

pedro.perez@ujat.mx

**** Universidad Juárez Autónoma de Tabasco (UJAT)

Calle Llamarada No. 221, Fracc. Lomas del Dorado

Villahermosa, Tabasco, México. C.P. 86153

Celular: 9933988448

hector.yris@ujat.mx

\section{RESUMEN}

El objetivo de este trabajo es evaluar los aspectos sintácticos y semánticos del contenido del componente: criterios de evaluación del aprendizaje para identificar oportunidades de mejora en la redacción sintáctica y semántica de este componente. Los criterios de evaluación es un componente del formato: Programa de Estudio, de las asignaturas escolares de la Universidad Juárez Autónoma de Tabasco (UJAT). La evaluación se ha realizado mediante la revisión y análisis de los contenidos incluidos en el componente, contrastados con las características y orientaciones de redacción y definición de criterios de evaluación, sugeridas por la literatura revisada. Las asignaturas escolares que se han seleccionado para evaluar pertenecen al Área de Formación General (AFG) y son comunes en todas las carreras profesionales. Los resultados observados a partir del análisis y la contrastación evidencian que los profesores involucrados en la elaboración, revisión y certificación de Programas de Estudio, en particular en el componente criterios de evaluación tienen una débil cultura para diseñar e implementar un método efectivo y consistente de evaluación del aprendizaje, lo cual conduce a prácticas evaluativas que no son congruentes con los contenidos, enfoques y propósitos del modelo educacional, o son insuficientes o superficiales. Tales prácticas suceden mayormente por la carencia de bases teóricas y técnicas en algunas fases del proceso evaluativo. Los profesores encontrarán dificultades para esta tarea si no tienen una formación didáctica adecuada para definir e implementar correctamente criterios de evaluación que permitan evaluar de manera efectiva el aprendizaje de los estudiantes.

Palabras clave: Programa de estudio, criterios de evaluación, modelo educacional, aprendizaje, método de evaluación, educación superior.

\section{ABSTRACT}

The objective of this work is to evaluate the syntactic and semantic aspects of the content of the component: learning evaluation criteria to identify opportunities for improvement in the syntactic and semantic writing of this component. The evaluation criteria is a component of the format: Study Program, of the school subjects of the Universidad Juárez Autónoma de Tabasco. The evaluation has been made through the review and analysis of the contents included in the component, contrasted with the characteristics and guidelines of writing and definition of evaluation criteria, suggested by the reviewed literature. The school subjects that have been selected for evaluation belong to the General Training Area and are common in all professional careers. The results observed from the analysis and the comparison show that teachers involved in the elaboration, revision and certification of study programs, particularly in the evaluation criteria component, 
Perspectivas Docentes 63

Los Criterios de Evaluación del Aprendizaje en la Educación Superior

The Evaluation Criteria of Learning in Higher Education

Abel Federico Pérez Hernández / Cristian Janet Méndez Sánchez ${ }^{\star *} /$ Pedro Pérez Arellano*** / Héctor Manuel Yris Whizar ${ }^{\star \star \star *}$

have a weak culture to design and implement an effective and consistent evaluation method of learning, which leads to evaluative practices that are not congruent with the contents, approaches and purposes of the educational model, or are insufficient or superficial. Such practices occur mostly due to the lack of theoretical and technical bases in some phases of the evaluative process. Teachers will find it difficult to do this task if they do not have adequate didactic training to correctly define and implement evaluation criteria that allow effective evaluation of student learning.

Keywords: Program of study, evaluation criteria, educational model, learning, evaluation method, higher education.

\section{INTRODUCCIÓN}

Ante los cambios en la concepción del aprendizaje y la enseñanza, es evidente la necesidad de impulsar una profunda transformación en la evaluación educativa. Se reconoce que si la práctica de evaluación no se modifica, los supuestos cambios pedagógicos en la enseñanza no tendrán ninguna repercusión ni en el sistema educativo ni en el alumnado. Tal cambio en la evaluación debe hacerse en un marco interpretativo que permita tomar una postura coherente frente a la problemática educativa, técnica, normativa, institucional y social que generalmente le acompañan (UJAT, 2005, p. 46).

En la actualidad, se insiste en la importancia de que el propósito de la evaluación en el aula sea mejorar el aprendizaje y desempeño de los alumnos mediante la creación constante de mejores oportunidades para aprender, a partir de los resultados que aquéllos obtienen en cada una de las evaluaciones que presentan durante un ciclo escolar. Significa dejar atrás el papel sancionador y el carácter exclusivamente conclusivo o sumativo de la evaluación de aprendizajes, por uno más interesado en conocer por qué los alumnos se equivocan o tienen fallas para que, una vez identificadas las causas, sea posible ayudarlos a superarlas (SEP, 2013, p. 8).

El presente trabajo tiene como objetivo evaluar los aspectos sintácticos y semánticos del contenido del componente: criterios de evaluación del aprendizaje para identificar oportunidades de mejora en la redacción sintáctica y semántica de este componente, incluido en el Formato de Programa de Estudio, de las asignaturas correspondientes al Área de Formación General (AFG), de las carreras profesionales de la Universidad Juárez Autónoma de Tabasco, para identificar oportunidades de mejora en la redacción sintáctica y semántica. Este campo de datos forma parte del formato de programa de estudio de todas las asignaturas, en todas las carreras profesionales de la UJAT.

El AFG (UJAT, 2005, p. 36) es el área que pretende lograr la comprensión del entorno y la construcción de conocimientos propicios para la integración a una disciplina. Se estructura por nueve asignaturas: Derechos Humanos, Cultura Ambiental, Pensamiento Matemático, Herramientas de Computación, Lectura y Redacción, Filosofía, Lengua Extranjera, Ética, Metodología, así como por las asignaturas de iniciación a la disciplina, que sirven de fundamento para todo conocimiento profesional. Son estas nueve asignaturas nuestro objeto de estudio en este trabajo. Sin embargo, la totalidad de los programas de estudio de todas las carreras profesionales pueden ser revisados para identificar oportunidades de mejora en su elaboración y redacción sintáctica y semántica, en el campo de datos abordado en este trabajo, en un contexto educativo.

Este trabajo no pretende ser una guía para la definición de los criterios de evaluación del aprendizaje sino solamente comparar el estado que guarda la redacción sintáctica y semántica de este campo de datos, en los programas de estudio revisados, con las orientaciones sugeridas en la literatura consultada. Es decir, identificar los criterios de evaluación incluidos en los programas de estudio y compararlos con las características sugeridas en la literatura revisada, para determinar si son pertinentes o no. También, no pretende ser un documento de reflexión sobre la importancia de los criterios de evaluación, ni tampoco del papel que juegan en el proceso de enseñanza y aprendizaje sino solamente se presenta con carácter orientativo y de apoyo, esperando que la información que en él se recoge sirva a todas las partes involucradas en la elaboración de los programas de estudio en las Universidades.

La evaluación es uno de los medios más importantes para el cambio y la innovación, ya que determina cómo y qué es lo que los estudiantes estudian. A pesar de ello los actuales sistemas y procedimientos de evaluación ponen el énfasis en el trabajo del profesorado antes que en el aprendizaje de los estudiantes. Hay una clara necesidad de repensar estos sistemas y procedimientos pasando de un sistema en el que el profesorado transmite unas calificaciones a uno en el que tanto profesores como estudiantes desarrollan sus habilidades evaluativas y, en consecuencia, sus competencias docentes y profesionales (Ministerio de Educación y Ciencia, 2007, p. 22). Este marco de reforma en el que se requiere a los estudiantes 
Perspectivas Docentes 63

Los Criterios de Evaluación del Aprendizaje en la Educación Superior

The Evaluation Criteria of Learning in Higher Education

Abel Federico Pérez Hernández $/$ Cristian Janet Méndez Sánchez ${ }^{* *} /$ Pedro Pérez Arellano*** / Héctor Manuel Yris Whizar***

enfrentarse a nuevos cambios y que apliquen el pensamiento crítico en el estudio de las diferentes materias exige nuevos métodos de evaluación que introduzcan estrategias de evaluación más comprensivas que las tradicionales en las aulas universitarias.

\section{LA EVALUACIÓN DE LOS APRENDIZAJES}

Uno de los obstáculos que con mayor frecuencia tienen las instituciones educativas para realizar un trabajo sistemático de evaluación del aprendizaje que consiste (Leyva, 2010, p. 1) en la falta de una cultura de la evaluación entre los actores del proceso educativo: alumnos, profesores y directivos, lo que conduce a prácticas evaluativas que no son congruentes con los contenidos, enfoques y propósitos del modelo educativo, o bien son insuficientes o superficiales. Tales prácticas suceden en gran medida por la carencia de bases teóricas y técnicas en algunas de las fases del proceso evaluativo: la planeación, el diseño y desarrollo de instrumentos de evaluación; el análisis de la información, o la interpretación y uso de los resultados. Estas carencias se reflejan en la emisión de juicios de valor subjetivos, superficiales o sesgados que impactan de manera negativa en los estudiantes.

La evaluación dentro del proceso enseñanza y aprendizaje (UJAT, 2005, p. 46) es una tarea de gran complejidad, porque le exige al docente analizar este proceso desde diversas perspectivas y enfrentarse a una serie de asuntos y problemas difíciles de abordar, de carácter psicopedagógico, técnicopráctico, administrativo-institucional y sociocultural. Es por ello que, una mejora en las propuestas de enseñanza y aprendizaje requiere necesariamente un cambio significativo en los modos de entender y realizar la evaluación.

Para García (2010) el proceso de evaluación debe estar íntimamente ligado a la determinación de los objetivos de la programación docente y los métodos, técnicas y recursos didácticos empleados. Por otro lado, la evaluación según (Villalobos, 2009, p. 148) necesita comprenderse como una oportunidad de aprendizaje. Asimismo, debe concebirse el error como fuente de conocimiento (de lo que no es o no se debe hacer) y como oportunidad de rectificar y, por ende, de aprender. La evaluación no debe considerarse como el valor simbólico del profesor; la evaluación precisa acompañarse indefectiblemente de la prudencia y de la justicia. Además, es necesario que el docente reflexione y comprenda su propia práctica de la evaluación, así como sus procesos de intervención pedagógica de la misma evaluación. Esta definición hace énfasis en la importancia de realizar un análisis profundo para identificar aquellas actividades de aprendizaje que han sido favorables para los alumnos y aquellas que no lo han sido, para corregir y aprender.

La manera de evaluar (Berry, 2010, p. 97) a los estudiantes influye directamente en su aprendizaje. Si un procedimiento de evaluación efectivo es desarrollado apropiadamente, la evaluación puede mejorar el aprendizaje del estudiante. Así pues el reto del profesorado universitario radica (Ministerio de Educación y Ciencia, 2007, p. 22) en implicar a los estudiantes en tareas de evaluación significativas para ellos y que contribuyan a su aprendizaje, de tal forma que lo más satisfactorio sea su experiencia de la educación superior. Además, la evaluación debe concebirse como un proceso que requiere un amplio fundamento teórico y no sólo empírico; los profesores deben reconocer que son los actores responsables de diseñar e implementar los mecanismos que se utilizarán en cada una de las etapas de este proceso descritas (López y Hinojosa, 2001).

Es necesario encontrar respuestas sustentables y consistentes para diversas preguntas propuestas (Villalobos, 2009, p. 148), tales como: ¿para qué evaluar?, es decir, la finalidad de la evaluación tomando en cuenta los objetivos educativos que se hayan planteado; ¿qué evaluar?, es decir, conceptos, conocimientos, habilidades, actitudes, considerando los contenidos educativos incluidos en las unidades de aprendizaje; ¿cómo evaluar?, a través del uso de estrategias didácticas: portafolios, rúbrica, organizadores gráficos, método de proyectos, etc. para evaluar no sólo aspectos cuantitativos sino cualitativos; ¿con qué evaluar?, esto es, los recursos didácticos en los cuales se incluyen aquellos criterios de evaluación que servirán como variables las cuales permitan de manera justa y objetiva asignar un valor numérico o una letra según sea el caso.

También (Suárez 1991, citado por Del Río, Candelas \& Farrand 2007) en su opinión sugiere que, antes de efectuar una evaluación, deberán responderse las siguientes interrogantes: ¿para qué se va a evaluar?, ¿qué se va a evaluar?, ¿a quién se va a evaluar?, ¿cuándo y dónde se va a evaluar?, ¿cómo se va a evaluar?, ¿Qué instrumentos se emplearán?, ¿cómo se van a analizar los datos? y ¿cómo se van a comunicar los resultados?. 
Perspectivas Docentes 63

Los Criterios de Evaluación del Aprendizaje en la Educación Superior

The Evaluation Criteria of Learning in Higher Education

Abel Federico Pérez Hernández $/$ Cristian Janet Méndez Sánchez ${ }^{\star *} /$ Pedro Pérez Arellano*** / Héctor Manuel Yris Whizar***

\section{CRITERIOS DE EVALUACIÓN DEL APRENDIZAJE}

Los criterios de evaluación (García, 2010, p. 81) son los principios, normas o ideas de valoración en relación a los cuales se emite un juicio valorativo sobre el objeto evaluado. Deben permitir entender qué conoce, comprende y sabe hacer el alumno, lo que exige una evaluación de sus conocimientos teóricos, su capacidad de resolución de problemas, sus habilidades orales y sociales, entre otros aspectos. Según esta autora, para definir los criterios de evaluación es necesario tener en cuenta las siguientes recomendaciones:

- Para cada contenido determinar que competencias se esperan desarrollar y establecerle un criterio de evaluación.

- $\quad$ Especificar claramente el tipo y grado de aprendizaje que se pretende que el alumno alcance. Deben hacer referencia a aprendizajes relevantes, entendiendo como tales, aquellos necesarios para que el alumno avance en dicho proceso.

- Determinar un aprendizaje mínimo y, a partir de él, fijar diferentes niveles para evaluar la diversidad de aprendizajes.

Esta autora menciona terminología interesante relacionada con los criterios de evaluación los cuales deben concretarse en distintas dimensiones, subdimensiones y atributos que permitan medir de manera más precisa la evolución en el aprendizaje de los alumnos, su nivel y calidad. Introduce el concepto de escalas de valoración para medir las dimensiones, subdimensiones y atributos asociados a cada tarea de evaluación. Ejemplifica con una rúbrica o matriz de valoración para evaluar el desempeño de los alumnos y la puntuación o escala que se aplicará a cada criterio diseñado. Así, se tiene como estrategia de evaluación la elaboración de proyectos y como criterios de evaluación: presentación y defensa oral, redacción, y contenido. Cada uno de estos criterios entonces contiene una serie de dimensiones, subdimensiones y atributos de ser necesario; para el primer criterio se tiene: estructura de la exposición, contenido de la exposición; para el segundo criterio: claridad, adecuación ortográfica y gramatical; y para el contenido: título (coherencia con objetivos y problema), introducción (presentación adecuada de la estructura del proyecto, idoneidad de las fuentes y bibliografía utilizada). Estas dimensiones contendrán de ser necesario subdimensiones, las cuales a su vez estarán integradas por atributos, esto con la finalidad de alcanzar un nivel de detalle que permita tener escalas de valoración justas, objetivas y transparentes.

Es conveniente que la definición de estos niveles garantice la consistencia, objetividad y transparencia del proceso de evaluación, de lo contrario se corre el riesgo de tener un proceso de evaluación deficiente y subjetivo. El profesor debe estar seguro de que todos los aspectos cualitativos y cuantitativos enunciados en los criterios sean fáciles de asignar un valor de acuerdo a la escala de valoración, y evitar la ambigüedad en éstos, para eliminar toda asignación numérica basada en la mera observación, que podría o no resultar arbitraria. El nivel de abstracción en las dimensiones y subdimensiones debe ser tan profundo (a detalle) como sea necesario para garantizar que cada criterio con características cualitativas permita fácilmente asignarle un valor numérico, eliminando en la medida de lo posible la subjetividad y discrecionalidad. Esto es, garantizar que los criterios de evaluación aplicados sobre un mismo producto o evidencia a ser evaluada, permitan obtener la misma valoración final, en distintos momentos o de distintos profesores.

Para García (2010, p. 84) la conjunción de todos los elementos que forman parte del sistema de evaluación diseñado debería sintetizarse en el documento Procedimiento de Evaluación. Por otro lado, (Garfolo, States y Arabia, 2015) se presentan una serie de instrumentos de puntuación tales como: listas de cotejo (checklists), escalas de calificación básica y rúbricas; también aborda el tema de los métodos de evaluación de los resultados de aprendizaje del estudiante; afirma que los resultados de aprendizaje necesitan estar enunciados como una actividad, producto, o desempeño que pueda medirse; éstos, deben reflejar habilidades del pensamiento de orden superior y ser significativos para la disciplina; este tema lo abordan ampliamente (Pérez, Méndez y Pérez, 2016) con resultados interesantes.

Para Moon (2004, p. 21) "los criterios de evaluación pueden ser desarrollados a partir de los objetivos de aprendizaje o de las tareas de evaluación, pero en cualquiera de los casos deberían relacionarse con los objetivos de aprendizaje".

Sin embargo, esto depende del enfoque utilizado para formular y estructurar los programas de estudio descrito en los modelos educativos de las instituciones, dado que para un enfoque de formación por competencias, los criterios de evaluación se formulan a partir de las competencias a desarrollar enunciadas. 
Perspectivas Docentes 63

Los Criterios de Evaluación del Aprendizaje en la Educación Superior

The Evaluation Criteria of Learning in Higher Education

Abel Federico Pérez Hernández / Cristian Janet Méndez Sánchez ${ }^{\star *}$ / Pedro Pérez Arellano*** / Héctor Manuel Yris Whizar***

De acuerdo con el Ministerio de Educación (2006) en el modelo de evaluación para el aprendizaje, los alumnos y alumnas saben con anticipación los criterios que serán usados para observar y evaluar lo que producen, eso es, lo que hacen frente a las tareas propuestas en aula. Dichos criterios son formulados por los docentes y son trabajados en aula con los estudiantes como parte de las actividades regulares de aprendizaje. Los criterios de evaluación reflejan los objetivos curriculares, tanto aquellos que se refieren a la comprensión de los conocimientos como aquellos que se refieren a determinadas habilidades y destrezas. Para cada criterio son elaborados descripciones de niveles de logro, constituyendo un continuo de calidad desde un desempeño más básico a uno de excelencia.

Además, la selección de los criterios de evaluación (UNESCO, 1988, p. 50) depende del contexto educativo: formación anterior de los estudiantes, método de enseñanza, modelo de evaluación. Entonces, los profesores están obligados académicamente a conocer a priori todos estos datos, antes de definir e implementar estrategias y criterios de evaluación del aprendizaje de los alumnos; de lo contrario, se tendrán métodos de evaluación fundamentados en el empirismo y la improvisación con características tradicionalistas.

\section{LISTA DE ÍTEMS REVISADOS Y EVALUADOS}

A continuación se presenta una lista de ítems, incluidos de manera explícita en los programas de estudio revisados (mencionados anteriormente) y otros seleccionados a partir de algunos rasgos semánticos identificados; estos ítems representan los criterios de evaluación para evaluar el aprendizaje de los estudiantes en las diversas asignaturas.

- Ítems incluidos de manera explícita:

- Participación en clase (2)

- Puntualidad y asistencia (3)

- Trabajo final (2)

- Portafolio de evidencia y participación

- Evaluación escrita

- Que el estudiante se conduzca de manera original y con criterio propio, en sus explicaciones escritas, exposiciones, debates y ensayos.

- Que el estudiante cumpla con los requerimientos propios de su rol, (Asistencia, puntualidad, orden, atención, concentración y participación) (4).

- Que el estudiante se conduzca de manera original, con criterio propio en todos los discursos escritos y orales de los resultados del aprendizaje.

- Congruencia entre las imágenes de los filósofos, sus nombres y sus concepciones de la realidad y del conocimiento, en relación con las ejemplificaciones y argumentaciones expuestas por los estudiantes en el álbum.

- Que cada estudiante sea consciente del significado filosófico que tiene su participación lingüística, comunicativa y social en la representación escénica.

- Que el estudiante se conduzca de manera original y con criterio propio en todos los discursos escritos y orales respecto de los mapas conceptuales, representación escénica, reportes descriptivos y explicativos y la síntesis individual.

- Que el estudiante se conduzca de manera consciente, original y con criterio propio, en los discursos escritos y orales con los que explique los mapas conceptuales y exponga sus ensayos explicativos y propositivos.

Ítems identificados con rasgos de criterios de evaluación:

- Se evaluará al alumno con un texto que reúna información y narración.

- Tomar en cuenta la determinación del tema, la organización de las ideas, sintaxis y ortografía.

- Exposición de problemas resueltos. Se privilegiará la exposición detallada de los procedimientos realizados para solucionar los problemas (4).

(El número dentro de los paréntesis indica el número de apariciones de los ítems replicados en una misma asignatura para evaluar las distintas unidades de aprendizaje)

Dado que los programas de estudio revisados en este trabajo han sido formulados en términos de resultados del aprendizaje y no por competencias, se han identificado y analizado aquellos criterios de evaluación, utilizados para evaluar los aprendizajes de los alumnos, para determinar si éstos cumplen con las características sintácticas y semánticas descritas en la literatura revisada. Algunos de los ítems incluidos de manera explícita en los programas de estudio son: participación en clase; trabajo final; portafolio de evidencia; evaluación escrita; que el estudiante cumpla con los requerimientos propios de 
Perspectivas Docentes 63

Los Criterios de Evaluación del Aprendizaje en la Educación Superior

The Evaluation Criteria of Learning in Higher Education

Abel Federico Pérez Hernández / Cristian Janet Méndez Sánchez ${ }^{* *} /$ Pedro Pérez Arellano ${ }^{* * *} /$ Héctor Manuel Yris Whizar ${ }^{* * *}$

su rol (asistencia, puntualidad, orden, atención, concentración y participación); que cada estudiante sea consciente del significado filosófico que tiene su participación lingüística, comunicativa y social en la representación escénica; que el estudiante se conduzca de manera original y con criterio propio en todos los discursos escritos y orales respecto de los mapas conceptuales, reportes descriptivos y explicativos y la síntesis individual.

Es fácil identificar que estos ítems guardan una estructura sintáctica y semántica de estrategias de evaluación y no de criterios de evaluación puesto que carecen de dimensiones y subdimensiones, que impide tener una matriz de valoración para evaluar el desempeño de los alumnos y anotar la puntuación o escala. Estos ítems sin lugar a dudas no están redactados en términos de criterios de evaluación; por lo tanto, el profesor encontrará dificultades para evaluar puntualmente el aprendizaje de los estudiantes, y éstos encontrarán dificultades para saber a qué aspectos, tanto cualitativos como cuantitativos se les asignará una valoración final.

\section{RESULTADOS}

De acuerdo con lo expuesto arriba, se han revisado y evaluado los contenidos del campo criterios de evaluación de los programas de estudio de las asignaturas pertenecientes al área de formación general en la UJAT.

Se han identificado un total de 25 ítems, incluidos como criterios de evaluación en las nueve asignaturas revisadas y evaluadas; esta evaluación ha consistido en comparar las características sintácticas y semánticas de los ítems con las orientaciones sugeridas en la literatura revisada, y determinar si éstos cumplen o no con lo establecido para un criterio de evaluación; de esto, se ha encontrado que sólo un $24 \%$ de los ítems (seis) cumple parcialmente (características semánticas) con lo sugerido en la literatura y un $76 \%$ está formulado totalmente de manera incorrecta, con deficiencias, propias de una falta de conocimiento para la definición e implementación de este campo de datos o componente.

Los seis ítems son:

- Se evaluará al alumno con un texto que reúna información y narración.

- Tomar en cuenta la determinación del tema, la organización de las ideas, sintaxis $y$ ortografía.

- Exposición de problemas resueltos, se privilegiará la exposición detallada de los procedimientos realizados para solucionar los problemas.

Este último se replica en cuatro unidades de aprendizaje de una misma asignatura, los otros dos pertenecen a una asignatura distinta.

En estos ítems se identifican algunos destellos de características de la semántica, es decir, del significado que se le atribuye a las palabras, pero carecen de la sintaxis de un criterio de evaluación, es decir, con dimensiones, subdimensiones y atributos.

También, se ha encontrado que un elevado $56 \%$ del total de los programas de estudio revisados no incluye criterios de evaluación del aprendizaje de los alumnos con los cuales se evalúan los aprendizajes; un $22 \%$ de los programas contiene parcialmente características para enunciar criterios de evaluación, y un 22\% contiene criterios de evaluación planteados totalmente de manera incorrecta. Estos resultados permiten conocer que el total de ítems identificados es menor al número de unidades de aprendizaje (36) incluidas en los nueve programas de estudio revisados; esto, por supuesto, numéricamente señala que se tienen unidades de aprendizaje sin criterios de evaluación y que, por lo tanto, no es posible emitir objetivamente un juicio valorativo sobre el objeto evaluado.

Estos resultados ampliamente exhiben la falta de conocimiento y experiencia por parte de los profesores para vincular los resultados del aprendizaje formulados para cada unidad con criterios de evaluación, teniendo como resultado programas de estudio con componentes débilmente acoplados. Un amplio porcentaje de ítems encontrados corresponden más a una estrategia de evaluación del aprendizaje que a los criterios que se utilizarán para ser evaluadas. Una respuesta consistente a ¿cómo evaluar? y ¿con qué evaluar? tales estrategias podría conducir a la definición de los criterios de evaluación del aprendizaje y a la obtención de una matriz de valoración, para registrar las notas finales.

\section{DISCUSIÓN}

Esta revisión y análisis ha permitido identificar que un 
Perspectivas Docentes 63

Los Criterios de Evaluación del Aprendizaje en la Educación Superior

The Evaluation Criteria of Learning in Higher Education

Abel Federico Pérez Hernández / Cristian Janet Méndez Sánchez ${ }^{\star \star} /$ Pedro Pérez Arellano*** / Héctor Manuel Yris Whizar ${ }^{\star \star \star \star}$

amplio porcentaje de los criterios de evaluación listados en los programas de estudio impiden, desde una perspectiva particular:

- Valorar el aprendizaje del alumno de manera efectiva.

- A docentes y alumnos, tener información específica acerca del proceso de enseñanza y de aprendizaje (SEP, 2013, p. 19).

- Recabar información sobre los diferentes aspectos evaluados.

- Evidenciar la amplitud y profundidad de los aprendizajes logrados, así como la funcionalidad y flexibilidad de los mismos (UJAT, 2005, p. 48).

- Orientar las acciones de evaluación para verificar el logro de los aprendizajes esperados y el desarrollo de competencias de cada alumno y del grupo. (SEP, 2013, p. 19)

- Comprobar si se han alcanzado los objetivos formulados o si se han desarrollado las competencias enunciadas.

- Aplicar un procedimiento de evaluación efectivo lo cual dificulta que la evaluación ayude en la mejora del aprendizaje del estudiante.

- Conocer deficiencias de aprendizaje para implementar mecanismos efectivos de retroalimentación.

- Promover aprendizajes profundos y significativos.

- Conocer por qué los alumnos se equivocan o tienen fallas para que, una vez identificadas las causas, sea posible ayudarlos a superarlas (SEP, 2013, p. 8).

- Que tanto profesores como estudiantes desarrollen sus habilidades evaluativas y, en consecuencia, sus competencias docentes y profesionales (Ministerio de Educación y Ciencia, 2007, p. 22).

- Promover mejoras en los métodos de enseñanzaaprendizaje.

- Que el docente reflexione y comprenda su propia práctica de la evaluación, así como sus procesos de intervención pedagógica de la misma evaluación (Villalobos, 2009, p. 148).

- Implicar a los estudiantes en tareas de evaluación significativas para ellos y que contribuyan a su aprendizaje, de tal forma que lo más satisfactorio sea su experiencia de la educación superior (Ministerio de Educación y Ciencia, 2007, p. 22).

- Entender qué conoce, comprende y sabe hacer el alumno (García, 2010, p. 81).

\section{CONCLUSIONES}

La evaluación del aprendizaje puede realizarse con distintos niveles de conocimiento y experiencia por parte del evaluador; cuenta mucho las características del profesor y de las actividades que lleva a cabo en este proceso. Los responsables de la elaboración de los programas de estudio deben considerar las orientaciones sugeridas en la literatura para la formulación del campo de datos o componente abordado en este trabajo, de tal manera que su contenido sea pertinente y cumpla con las orientaciones. La ausencia de una característica obligatoria en la definición e implementación de criterios de evaluación, permite identificar que éstos necesitan una corrección para que sean concretos y no ambiguos.

El desconocimiento de la teoría pedagógica para realizar un completo proceso de evaluación del aprendizaje trae consigo consecuencias indeseables que seguramente impedirán al maestro: aplicar un proceso de evaluación consistente y eficaz; obtener evidencias para conocer los logros y avances del aprendizaje de los alumnos; detectar a tiempo problemas en el progreso del alumno; identificar y analizar las causas de los aprendizajes no logrados y tomar decisiones de manera oportuna; descubrir lo que el estudiante sabe o puede hacer; determinar el aprovechamiento de los estudiantes y tomar las decisiones pertinentes para la mejora a través de mecanismos efectivos de retroalimentación; valorar el nivel de desempeño y el logro de los aprendizajes esperados; conocer por qué los alumnos se equivocan o tienen fallas para ayudarlos a superarlas.

También, esto, seguramente impedirá al alumno: sentirse participante activo en su propia evaluación; aprender a evaluar sus propias acciones y aprendizajes, y reflexionar sobre los resultados; conocer con anticipación los criterios que serán utilizadas para valorar su aprendizaje. Estas carencias de bases teóricas y técnicas en algunas de las fases del proceso evaluativo seguramente se verán reflejadas en la emisión de juicios de valor subjetivos, superficiales o sesgados que impactan de manera negativa en los estudiantes. Este proceso de revisión y evaluación es aplicable a cualquier programa 
Perspectivas Docentes 63

Los Criterios de Evaluación del Aprendizaje en la Educación Superior

The Evaluation Criteria of Learning in Higher Education

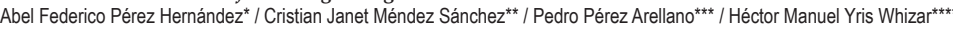

de estudio que incluya como componente al campo de datos criterios de evaluación, en un contexto educativo; también, es aplicable a cualquier proceso de evaluación de los aprendizajes realizado por los profesores, en un contexto de la educación superior.

Es recomendable que el profesor cuente con un pleno conocimiento y entendimiento de los métodos y técnicas de evaluación existentes en el ámbito docente actual, además de las cuestiones relativas a la finalidad de la evaluación. Si la práctica de evaluación no se modifica, los supuestos cambios pedagógicos en la enseñanza no tendrán ninguna repercusión ni en el sistema educativo ni en el alumnado. Es el maestro, quien con base en los objetivos, enfoques y propósitos del modelo educativo define y decide las técnicas e instrumentos que se usarán en el proceso de evaluación, y éste uso deberá estar documentado con claridad y objetividad. El proceso de diseño de criterios puede contribuir a asegurar que existe una vinculación clara entre el propósito del curso y los resultados que se prevén para los estudiantes.

De manera particular se considera que el componente criterios de evaluación no debería formar parte del formato de programa de estudio de las asignaturas pues es un campo que requiere de una definición y formulación amplia y estructurada en los términos descritos en secciones anteriores. En este trabajo no se cuestiona el papel que juega el proceso de evaluación en el aprendizaje de los estudiantes sino la forma en como este componente ha sido enunciado, y además aprobado para ser utilizado en todas las carreras profesionales ofertadas en la institución.

\section{SEMBLANZA DE LOS AUTORES:}

\section{Abel Federico Pérez Hernández}

Profesor Investigador en la Universidad Juárez Autónoma de Tabasco (UJAT), México, desde 2005; adscrito a la División Académica Multidisciplinaria de Jalpa de Méndez (DAMJM) desde 2014. Doctor en Sistemas Computacionales por la Universidad del Sur, Tuxtla Gutiérrez, Chiapas. Ha asistido a diversos diplomados en Formación de Tutores, Competencias Docentes en la Educación Superior y en Innovación Pedagógica. Ha impartido cursos en licenciatura, ha presentado ponencias en congresos internacionales de Informática y Sistemas y en foros de Innovación Educativa organizados por la UJAT. Ha publicado artículos en libros electrónicos y revistas especializadas en el campo de la educación superior.

\section{Cristian Janet Méndez Sánchez}

Egresada de la Licenciatura en Psicología por la Universidad Popular de la Chontalpa (UPCH), ubicada en la ciudad de Cárdenas, Tabasco, México. Tiene especial interés en temas de enfoques y estilos de aprendizaje, psicología educativa, métodos de enseñanza y estrategias para el aprendizaje.

\section{Pedro Pérez Arellano}

Profesor Investigador en la División Académica de Informática y Sistemas (DAIS) de la Universidad Juárez Autónoma de Tabasco (UJAT), México, desde 1989. Maestro en Docencia por la UJAT, Villahermosa, Tabasco. Ha asistido a diversos diplomados en Formación de Tutores y Competencias Docentes en la Educación Superior. Ha impartido cursos en licenciatura, ha presentado ponencias en congresos internacionales de Informática y Sistemas y en foros de Innovación Educativa organizados por la UJAT. Ha publicado artículos en libros electrónicos y revistas especializadas en el campo de la educación superior. Profesor con reconocimiento del Perfil PRODEP y miembro del Sistema Estatal de Investigadores.

\section{Héctor Manuel Yris Whizar}

Profesor Investigador en la División Académica de Informática y Sistemas (DAIS) de la Universidad Juárez Autónoma de Tabasco (UJAT), México. Maestro en Gestión de Tecnologías de la Información por la Universidad Tec Milenio, Villahermosa, Tabasco. Ha asistido a diversos diplomados en Formación de Tutores, Competencias Docentes en la Educación Superior. $\mathrm{Ha}$ impartido cursos en licenciatura, ha presentado ponencias en congresos internacionales de Informática y Sistemas y en foros de Innovación Educativa. Ha publicado artículos en libros electrónicos. Profesor con reconocimiento del Perfil PRODEP y miembro del Sistema Estatal de Investigadores. 
Perspectivas Docentes 63

Los Criterios de Evaluación del Aprendizaje en la Educación Superior

The Evaluation Criteria of Learning in Higher Education

Abel Federico Pérez Hernández / Cristian Janet Méndez Sánchez ${ }^{\star \star} /$ Pedro Pérez Arellano*** / Héctor Manuel Yris Whizar***

\section{REFERENCIAS BIBLIOGRÁFICAS:}

Del Río, F., Candelas, M., \& Farrand, J. (2007, julio-diciembre). Diseño de Estrategias de Aprendizaje con Enfoque en el Proceso Administrativo. Revista Mexicana de Agronegocios, XI, pp. 425-434.

Díaz, F. (2006). Enseñanza situada: vínculo entre la escuela y la vida. México: McGraw-Hill.

European Agency. Assessment for Learning and Pupils with Special Educational Needs. Recuperado de https:// www.european-agency.org/sites/default/files/assessmentfor-learning-and-pupils-with-special-educational-needs assessment-for-learning-graphic-en.pdf.

García, I. (2010). Sistema de evaluación. Universidad de Salamanca.

Garfolo, B. T., States, U., \& Arabia, S. (2015). Demystifying Assessment. Journal of College Teaching \& Learning. 12(4), 151-170. Recuperado de http://files.eric.ed.gov/fulltext/ EJ1067282.pdf.

Leyva, Y. E. (2010). Evaluación del aprendizaje: Una guía práctica para profesores. Recuperado de http://www.ses.unam. mx/curso2012/pdf/Guia_evaluacion_aprendizaje2010.pdf.

López, B. S. \& Hinojosa, E. M. (2001). Evaluación del aprendizaje: alternativas y nuevos desarrollos. México: Trillas.

Ministerio de Educación. (2006). Evaluación para el aprendizaje: Enfoque y materiales prácticos para lograr que sus estudiantes aprendan más y mejor. Chile. Recuperado de http://portales.mineduc.cl/usuarios/ cpeip/File/2013materialespsp/mediaepa.pdf.

Ministerio de Educación y Ciencia. (2007). Proyecto: EvalCOMIX: Evaluación de competencias en un contexto de aprendizaje mixto (BlendedLearning). España. Recuperado de http://tecnologiaedu. us.es $/ \mathrm{mec} 2011 / \mathrm{htm} / \mathrm{mas} / 5 / 51 / 19$.pdf.

Moon, J. (2004). Linking levels, learning outcomes and assessment criteria. In Edinburgh. Recuperado de http://scholar.
google.com/scholar?hl=en\&btnG=Search\&q=intitle:Linking+Le vels,+Learning+Outcomes+and+Assessment+Criteria\#1.

Pérez, A. F., Méndez, C. J. \& Pérez, P. (2016). The learning outcomes in the study program format of school subjects belonging to the General Training Area at UJAT: A syntactic and semantic analysis. Perspectivas docentes, 26(60), 15- 20.

SEP. (2013). Secretaría de Educación Pública. Las estrategias y lo instrumentos de evaluación desde el enfoque formativo. En serie: Herramientas para la evaluación en educación básica (p. 13). México.

UJAT. (2005). Modelo educativo. Universidad Juárez Autónoma de Tabasco.

UNESCO. (1988). Directrices para la redacción de objetivos de aprendizaje en bibliotecología, ciencias de la información y archivística. Paris.

Villalobos, M. (2009). Evaluación del aprendizaje basado en competencias. México: Minos Tercer Milenio. 